\title{
Research of an Embedded IOT Gateway Applied to Post-earthquake Rescue
}

\author{
http://dx.doi.org/10.3991/ijoe.v11i9.5065 \\ Lili Xing ${ }^{1}$, Bing Zhang* ${ }^{1,2}$, Qiong $\mathrm{Wu}^{2}, \mathrm{Li} \mathrm{Xie}^{1}$ \\ ${ }^{1}$ Institute of Disaster Prevention, Yanjiao Town, Sanhe City, Hebei Province, China \\ ${ }^{2}$ Institute of Geophysics, China Earthquake Administration, Haidian District, Beijing, China
}

\begin{abstract}
The wireless self-organization network, such as Zigbee network typically can be constructed quickly to connect the post-earthquake field and the command center. So, an IOT (Internet of Things) gateway is the necessary device to connect Zigbee network to TCP/IP network. In this paper, an embedded IOT gateway based on Xscale PXA270 was designed to send the data of demands such as drugs, food, excavators, reinforce from post-earthquake field to the command center to enhance the rescue efficiency. The experimental results show that the gateway has a high performance and can be applied to postearthquake rescue.
\end{abstract}

Index Terms-Internet of Things, Gateway, Postearthquake Rescue.

\section{INTRODUCTION}

After earthquake, various kinds of emergency wireless communication networks would be constructed in the post-earthquake rescue scene. For example, there is a deployment system of earthquake relief based on Zigbee that was developed by the first author of this paper. The system above consists of hand-held terminals and command centre. The former sends the signal of materials demands to the command centre through the wireless selforganized $2.4 \mathrm{GHz}$ Zigbee network. The command centre deploys the relief supplies according to the received demands, meanwhile, sends the confirmation signal to the terminal. An Internet of Things (IOT) gateway is needed to transmit the demand signal to command centre [1]. Additionally, because different networks have different working frequencies, different working modes, and different protocols, how to ensure the communication between different emergency wireless networks and the command center LAN is a key problem.

In response to the problem, an IOT (Internet of Things) gateway that can fast communicate with Zigbee and WCDMA (Wideband Code Division Multiple Access) was developed based on Xscale PXA270. The test proved that the gateway can transmit the rescue data quickly in time and enhance the rescue efficiency [2].

\section{DESIGN OF THE SYSTEM STRUCTURE}

The system structure is shown in figure 1 . In the postearthquake rescue scene, the wireless network can be Zigbee or WCDMA, which connected to the embedded IOT gateway to provide the demand information and environment data. As server, the IOT gateway catches the data from the wireless network and stores it into database. At the same time the data will be transferred to web server application according to different service such as the demand information and environment data. Additionally, the web server receives the HTTP requires from remote terminals which are connected to the gateway through $\mathrm{TCP} / \mathrm{IP}$ protocol. Then the web server will transfer the data that the remote terminals require to the correspondent terminal through TCP/IP protocol.

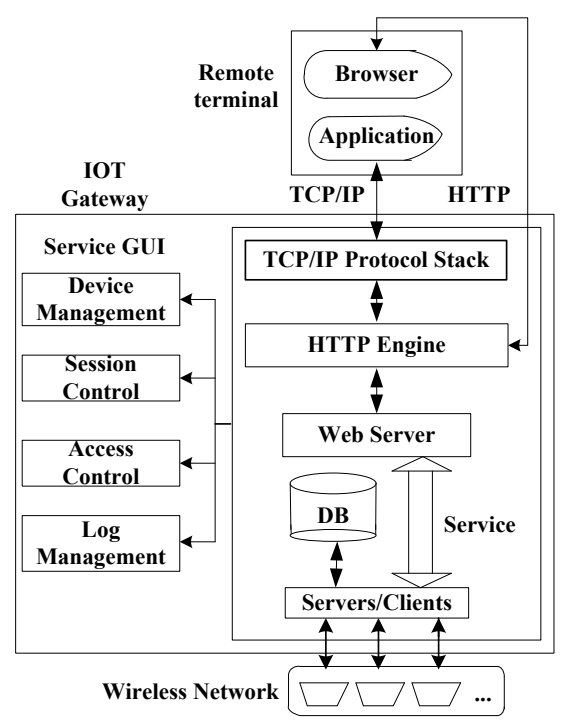

Figure 1. System Structure

Additionally, the gateway can be managed through service GUI (Graphical User Interface), such as device management which can set the work mode of hardware, session control which can control the begin and finish of sessions, access control which can set the IP address which can be connected to the gateway, log management which can manage all of the operations. The remote terminals can browser the data from the wireless network in the post-earthquake rescue scene and control the terminals in the wireless network through IE browser [3].

\section{DESIGN OF THE IOT GATEWAY}

\section{A. The function design}

The functions of the gateway are network nodes state management, network nodes control management, data requiring nodes management, network nodes data acquisition management, data imaging show and.

The gateway receives the demands information and displays all the network nodes and their father nodes. The selected nodes information includes RSSI ( Received 
Signal Strength Indicator), address and data. Network nodes control management takes charge of sending control command to network nodes to reboot the network nodes. Data requiring nodes management is in charge of the show and control of data requiring nodes. Network nodes data acquisition management implements the functions of data acquisition, processing and show. Data imaging show management format the data to display on LCD screen. Feedback signal management can control the LED and buzzer on the terminal in wireless network.

\section{B. Hardware design of IOT gateway}

The hardware structure of IOT gateway is shown as figure 2. As microprocessor, PXA270 executes program, process data and control periphery devices. As memory, SDRAM DDR stores temporarily the running software such as Windows Embedded CE operating system, hardware drivers, network protocol and application software. FLASH is used to store Windows Embedded CE and the bootloader which can initialize hardware and build memory space mapping. TFT LCD is used to humancomputer interaction. SD CARD is used to store the data acquired from wireless network nodes. USB is used to connect the WCDMA or IPv6 network modules. RS232 is used to connect the converged node of wireless Zigbee network. JTAG is used to system debug. Ethernet Controller is used to connect TCP/IP network [4].

The PXA270 processor is the first Intel processor to include Intel Wireless MMX technology, enabling highperformance, low-power multimedia acceleration with a general-purpose instruction set. Intel Quick Capture technology provides a flexible and powerful camera interface for capturing digital images and video. While performance is key in the PXA270 processor, power consumption is also a critical component. The new capabilities of Wireless Intel SpeedStep technology set the standard for low-power consumption.

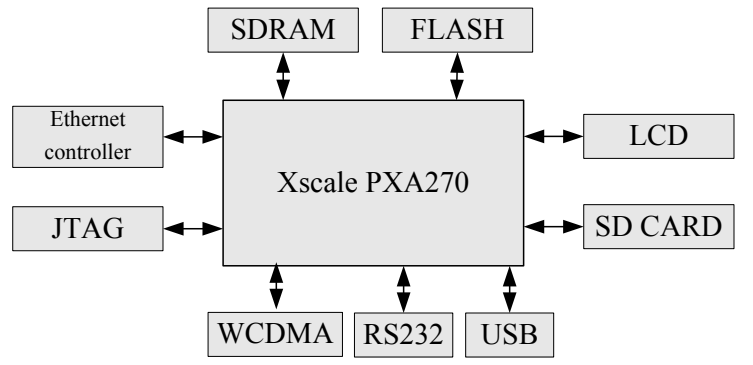

Figure 2. IOT Gateway Hardware Structure

The wireless Zigbee module, whose core is CC2430 chip, is connected to the microprocessor Xscale PXA270 by CON1 port. The module includes MCU/RF, power management, microsensors and external interfaces. The overall framework is just as the figure 3. MCU CC2430 is not only the core processor but also the $2.4 \mathrm{G}$ RF wireless transceiver, which is equipped with $32 \mathrm{MHz}$ 和 32.768 $\mathrm{KHz}$ crystal oscillators, six LED indicator lights, and eight buttons. Additionally, the microsensors such as Temperature and humidity are connected to the CC2430 through I/O port. The photoelectric and voltage signals are captured through the internal AD. The voltage of the whole module is DC $3.3 \mathrm{~V}$, and the chip internal voltage is DC $1.8 \mathrm{~V}$.

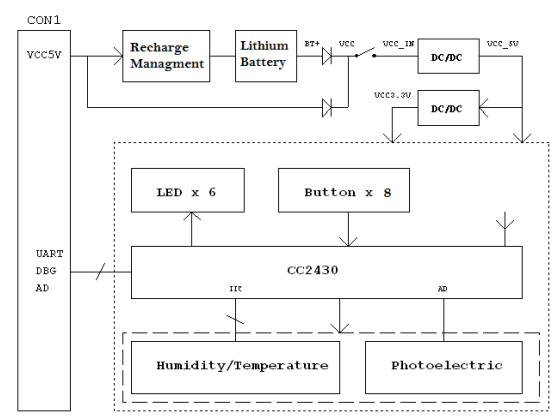

Figure 3. Zigbee module structure chart

When the processor PXA270 is connected to ghe Zigbee module CC2430 and exchanges data, the communication baud rate needs to be set. There is an internal baud rate generator that sets the UART baud rate when operating in UART mode in theCC2430. The UxBAUD.BAUD_M [7:0] and UxGCR.BAUD_E[4:0] registers define the baud rate used for UART transfers. The baud rate is given by the following equation:

$$
\text { Baudrate }=\frac{\left(256+B A U D_{-} \mathrm{M}\right) \times 2^{B A U D_{-} E}}{2^{28}} \times F
$$

$\mathrm{F}$ is the system clock frequency, $32 \mathrm{MHz}$ in the equation 1 .

The register values required for standard baud rates are shown in Table 1 for a typical system clock set to 32 MHz. Table 1 gives the difference in actual baud rate to standard baud rate value as a percentage error. The maximum baud rate for UART mode is $\mathrm{F} / 16$ when BAUD_E is 16 and BAUD_M is 0, and where $\mathrm{F}$ is the system clock frequency.

TABLE I.

BAUD RATE SETTINGS FOR 32 MHZ SYSTEM CLOCK

\begin{tabular}{|c|c|c|c|}
\hline $\begin{array}{c}\text { Baud rate } \\
\text { (bps) }\end{array}$ & $\begin{array}{c}\text { UxBAUD.BAUD_ } \\
\mathbf{M}\end{array}$ & UxGCR.BAUD_E & Error(\%) \\
\hline 2400 & 59 & 6 & 0.14 \\
\hline 4800 & 59 & 7 & 0.14 \\
\hline 9600 & 59 & 8 & 0.14 \\
\hline 14400 & 216 & 8 & 0.03 \\
\hline 19200 & 59 & 9 & 0.14 \\
\hline 28800 & 216 & 9 & 0.03 \\
\hline 38400 & 59 & 10 & 0.14 \\
\hline 57600 & 216 & 10 & 0.03 \\
\hline 76800 & 59 & 11 & 0.14 \\
\hline 115200 & 216 & 11 & 0.03 \\
\hline 230400 & 216 & 12 & 0.03 \\
\hline
\end{tabular}

C. Software design of IOT gateway

The hierarchy method is applied to the design of the hand-held terminal software. The software hierarchy is shown in figure 4 . The layers of gateway software are operating system, hardware driver, application and web server from bottom to top. Windows Embedded CE takes charge in the management of programs of all of layers. On the one hand, it manages the load of drivers, memory allocation and recycling. On the other hand, it provides communication interface, data sharing and threads management for the calls between programs of different layers. The hardware drivers are in charge of the working of all of hardware. The application implements the functions that introduced in section 3.1. The web server 
will produce different web pages according to the application so that the remote terminals can browser the information through IE [5].

\begin{tabular}{|c|c|}
\hline \multicolumn{1}{|c|}{ Gateway Software } & Remote Terminal Software \\
\hline Web Server & IE Browser \\
\hline Application & \\
\hline Hardware Driver & Desktop Operating System \\
\hline Windows Embedded CE & \\
&
\end{tabular}

Figure 4. FIGURE 4 IOT Gateway Software Hierarchical Structure

\section{1) Data-processing software design of gateway}

When the gateway receives data from the wireless network nodes, the data-processing application starts and get ready for the link to the upper application. All the nodes connect to the coordinator which sends the received information to the remote terminals. At the same time, upper application enters the message loop. Through calling MyOnReceive function, the upper application reacts differently according to the different information codes [6]. PC sends feedback information through controlling the LED and the buzzer after receiving the demand information. The flow chart is shown as Figure 5.

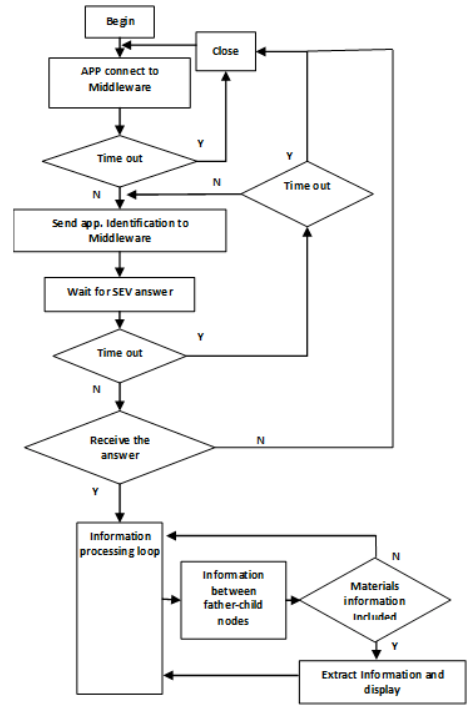

Figure 5. IOT Gateway Data Processing Flow Chart

The processed data is transmitted from the Zigbee module to the PXA270 through a protocol that is a data structural body in program. The structural body is as follow.

typedef struct

\{

uint8 start;

uint16 group;

uint 16 address;

uint8 exist;

uint8 type;

uint8 name;

float data[4];

uint8 mac[8];

uint8 pmac[8];

uint 8 buzzer;

uint8 ledstate[8];

unit8 end;

\}NodePCDataPacket_t;

$/ /$ Head $/ /$ network group ID

// Node Address

//in the network or not

//network type

//sensor type

// sensor data

//MAC address

//MAC address of parent node //buzzer

$/ /$ LED states

$/ /$ End

The control command is transmitted from the PXA270 to the Zigbee module through a protocol that is a data structural body in program. The structural body is as follow.

$\begin{array}{lc}\text { typedef struct } & \\ \text { uint8 start; } & \text { //Head } \\ \text { uint16 group; } & / / \text { network group ID } \\ \text { uint16 address; } & / / \text { Node Address } \\ \text { uint8 type; } & / / \text { network type } \\ \text { uint8 name; } & / / \text { sensor type } \\ \text { uint8 cmd[4]; } & / / \text { control commands } \\ \text { unit8 end; } & \text { //End }\end{array}$

\}PCNodeCmdPacket_t;

\section{2) Web server program design of gateway}

There is web server application in Windows Embedded $\mathrm{CE}$ that listening on port 80 . Once there is a data require, the web server application will receive a HTTP request signal. The gateway will quickly retrieve the real-time data from the database and make an updated HTML web page. For processing a request, the web server would either response a static webpage or an image to redirect a new web page or delegate the dynamic response to another application, JSP (JavaServer Pages), servlets, ASP (Active Server Pages), server-side JavaScript or other server-side technology. Either of the server-side applications typically produce a HTML response, so that the remote terminals can browser the information through IE $[7,8]$. The web service program flow is shown as figure 6.

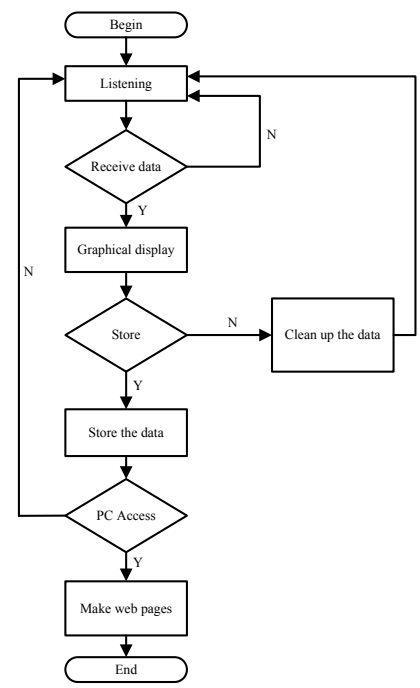

Figure 6. IOT Gateway Web Service Program Flow Chart

\section{SYSTEM TESTING RESULT}

In order to test the gateway, a 100M LAN were build with 16 computers and the designed gateway. Additionally, 8 Zigbee network nodes and 1 WCDMA module were connected to the gateway. Then WebBench testing software was used to test the performance index of gateway, such as the processing requests per second (prpe), CPU usage rate (CPUus), memory footprint size (mfs) and loading time (lt), when there were 1, 2, 4, 8, 16 computers accessing the gateway. The average numbers of test computers were 6.2 as the figure 7 shown. The average processing requests per second was 7.4. The average CPU usage rate was $8.04 \%$. The average memory footprint size was $97.8 \mathrm{~KB}$. The average loading time was 
$8.2 \mathrm{~ms}$. The test results show that the designed gateway has a high performance and can be applied to post-earthquake rescue.

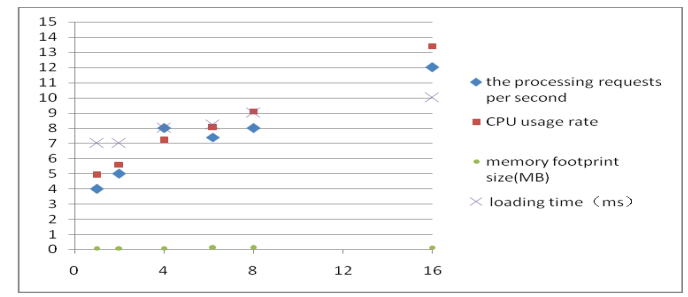

Figure 7. IOT Gateway Performance Test Result

\section{CONCLUSIONS}

Belonging to network layer in the architecture of IOT, the gateway transmit the demand signal from the former handheld terminal through $2.4 \mathrm{GHz}$ Zigbee protocol to the command center through TCP/IP protocol.

From the test above, we can draw the following conclusions. The IOT gateway would not crash when it continued working without poweroff, with high speed and capability. Also, it can enhance the rescue efficiency. But there is also a lot of work to further do. For example, the networks of TD-SCDMA (Time Division-Synchronization Code Division Multiple Access) and CDMA2000 should be integrated into the gateway.

\section{ACKNOWLEDGMENT}

I'd like to express my sincere thanks to Scientific Research Plan Projects for Higher Schools in Hebei Province (Z2015111) and Earthquake Science and Technology Spark Plan, China Earthquake Administration (XH15066Y) that sponsor the paper.

\section{REFERENCES}

[1] ZHANG Bing, XING Lili, YANG Fan. (2013). A DEPLOYMENT SYSTEM OF EARTHQUAKE RELIEF SUPPLIES BASED ON ZIGBEE. Journal of Theoretical and Applied Information Technology, 55 (3): 673-676.

[2] XU Jinghai, YANG Yan, DENG Minxian. (2010). GIS-based Quick Report and Estimation of Earthquake Disaster Information. Journal of Natural Disasters, 19(4): 141-146.

[3] Beijing Seismological Bureau. (2010-8-30). The research work about Internet of Things applications to the Beijing Seismological Bureau by Zhou Zhengyu, Deputy Secretary-General of Beijing Municipal Government. http://www.cea.gov.cn/manage/html/8a8587881632fa5c0116674a 018300cf/content/10_08/30/1283158124444.html.

[4] SUN Xu-guang, HUANG Meng, SHAN Wei-feng, ZHANG Xing. (2010). Design of event-triggered monitoring system based on wireless sensor networks. Computer Engineering and Design, 31(4): 744-746.

[5] Yang Linnan, Li Honggang. (2008). Research of Embedded Web Server Based on ARM9. Computer Measurement \& Control, 16(12): 1939-1942.

[6] ZigBee Standard Organization. (2008-05-06). ZigBee2007 Protocol Specifications [EB/OL]. http://www.sikaitech.com/kaifaziliao/ZigBee2007/18337694.html.

[7] Microsoft. (2010-06-01). SDK Development (Windows Embedded CE 6.0). http://technet.microsoft.com/zhcn/library/ee482195.

[8] Michael Barr. (1999). Programming Embedded Systems in C and $\mathrm{C}++$, O’Reilly \& Associates Inc.,

\section{AUTHORS}

Bing Zhang (Corresponding Author), Qufu City, Shandong Province, China. A full-time teacher from 2008 in China Institute of Disaster Prevention, lecturer. University studies: Computer Application. Scientific interest: Internet of Things. Publications: 6 papers. Email: zhangbing@cidp.edu.cn

Lili Xing, Tangshan City, Hebei Province, China. A full-time teacher from 2008 in China Institute of Disaster Prevention, lecturer. University studies: Computer Application. Scientific interest: Data Mining. Publications: 6 papers. Email: xinglili@cidp.edu.cn

Qiong Wu, Changge City, Henan Province, China. Assistant Researcher, Institute of Geophysics, China Earthquake Administration, from 2011 until now, Ph. d. University studies: Electrical Engineering and Automation. Scientific interest: Geophysical Instrumentation. Publications: 8 papers. Email:wuqiong_cea@hotmail.com

Li Xie, Tianshui City, Gansu Province, China. A fulltime teacher from 2005 in China Institute of Disaster Prevention, lecturer. University studies: Beijing University of Technology. Scientific interest: Computer application. Publications: 5papers. Email: xieli@cidp.edu.cn

This work was financially supported by the Scientific Research Plan Projects for Higher Schools in Hebei Province (Z2015111) and Earthquake Science and Technology Spark Plan, China Earthquake Administration (XH15066Y). Submitted 21 September 2015. Published as resubmitted by the authors 20 October 2015. 